\title{
THE EICHLER COHOMOLOGY GROUPS AND AUTOMORPHIC FORMS
}

\author{
BY \\ R. C. GUNNING( $\left.{ }^{1}\right)$
}

Introduction. In his papers $[5 ; 6]$ Eichler demonstrated the significance for the study of automorphic forms of Bol's discovery [3] of some remarkably simple differential operators taking automorphic forms into automorphic forms. In [6] in particular Eichler discussed a relation between the automorphic forms associated to a transformation group $\mathcal{G}$ on a Riemann surface $D$ and some purely algebraic constructions involving the group $\mathcal{G}$, the first cohomology groups of $\mathcal{G}$ with certain modules of polynomials as coefficients; the cocycles appeared as the periods of the automorphic forms under iterated indefinite integration, generalizing the classical interpretation of the periods of the abelian integrals on $D / G$ (which can of course be considered as automorphic forms on $D$ ) as cocycles of the group $\mathcal{G}$ or alternatively of the space $D / G$. The object of studying such a relation is the development of tools for calculating the dimensions of spaces of automorphic forms and the traces of the Hecke operators on automorphic forms. The aim of the present paper is the study of a more general form of this relation in somewhat greater detail for one complex variable, but in such a manner that the results can be extended to several complex variables; the actual extension to several complex variables, as well as the application to the study of the Hecke operators, will be discussed elsewhere.

As for the contents of this paper, $\S 1$ is devoted to an exposition of Bol's differential operators in a form more useful in the present context than that of [3]. In $\$ 2$ these differential operators are applied to give an exact cohomology sequence containing, in a rather more transparent form, the relation of Eichler discussed above. The interpretations of the terms appearing in this exact sequence are discussed in $\$ \S 3$ through 5 ; the only point of difficulty arises in $\S 4$, Theorem 3 of that section really being a form of the Serre duality theorem [12] appropriate to the occasion. These results are combined in $\$ 6$ to give a formal statement of the fundamental result of the paper.

1. Differential operators preserving automorphic forms. Let $\mathfrak{H C}$ be the group of $2 \times 2$ real matrices

$$
T=\left(\begin{array}{ll}
a & b \\
c & d
\end{array}\right)
$$

Received by the editors August 24, 1960 .

(1) The author holds an Alfred P. Sloan Fellowship. 
such that $\Delta_{T}=a d-b c>0 ; \mathfrak{H}$ acts as a group of complex analytic automorphisms of the upper half plane $D=\{z \in C \mid \operatorname{Im} z>0\}$, with the group action defined by $T: z \rightarrow T z=(a z+b) /(c z+d)$. A scalar multiplier $\rho(T, z)$ for the group $\mathcal{H C}$ acting on $\mathfrak{D}$ is a complex-valued function on $\mathcal{H} \times \mathbb{D}$ which is holomorphic as a function on $D$ and which satisfies the functional equations $\rho\left(T_{1} T_{2}, z\right)=\rho\left(T_{1}, T_{2} z\right) \rho\left(T_{2}, z\right)$ for all $T_{1}, T_{2} \in \mathcal{H}$. The classification of such multipliers has been discussed elsewhere [9]; for the present purposes only multipliers of the form

$$
\rho(T, z)=\Delta_{T}^{-k / 2}(c z+d)^{k}
$$

will be considered, where $k$ is a positive or negative integer called the degree of the multiplier and the positive square root of $\Delta_{T}$ is selected in each case. As a notational convenience the scalar multiplier of degree $k=2$ will be denoted by $\delta(T, z)$; thus $\delta(T, z)^{-1}=d T(z) / d z$. Note that any product of multipliers, such as $(\rho \delta)(T, z)=\rho(T, z) \delta(T, z)$, is again a multiplier.

Having selected a multiplier $\rho$, associate to each complex-valued function $f$ on $D$ and each transformation $T \in \mathcal{F}$ the complex-valued function $\left.f\right|_{\rho} T$ on D defined by

$$
\left(\left.f\right|_{\rho} T\right)(z)=\rho(T, z)^{-1} f(T z) .
$$

It is clear that $\left.\left(a_{1} f_{1}+a_{2} f_{2}\right)\right|_{\rho} T=a_{1}\left(\left.f_{1}\right|_{\rho} T\right)+a_{2}\left(\left.f_{2}\right|_{\rho} T\right)$ for any constants $a_{1}, a_{2}$ and functions $f_{1}, f_{2}$; and that $\left.f\right|_{\rho}\left(T_{1} T_{2}\right)=\left.\left(\left.f\right|_{\rho} T_{1}\right)\right|_{\rho} T_{2}$ for any transformations $T_{1}, T_{2} \in \mathcal{H}$. The mapping $\left.(f, T) \rightarrow f\right|_{\rho} T$ is therefore a representation of $\mathcal{H C}$ as a group of linear transformations on, say, the vector space $\sqrt{S}$ of $C^{\infty}$ complexvalued functions on $D$; this representation will be denoted by $\rho$ as well.

A linear differential operator is also a linear transformation on the vector space $\mathfrak{S}$; following Eichler the differential operators of interest are those which commute with the representation $\rho$ of $\mathscr{H}$ on $\mathfrak{C}$, in an appropriate sense. As usual when considering holomorphic functions introduce as generators of the algebra of linear differential operators the first order operators

$$
\frac{\partial}{\partial z}=\frac{1}{2}\left(\frac{\partial}{\partial x}-i \frac{\partial}{\partial y}\right) \text { and } \frac{\partial}{\partial \bar{z}}=\frac{1}{2}\left(\frac{\partial}{\partial x}+i \frac{\partial}{\partial y}\right)
$$

where $z=x+i y ;$ and put

$$
\mathfrak{D}_{\rho}=\frac{\partial}{\partial z}+\frac{k}{2 i y}
$$

where $k$ is the degree of the multiplier $\rho$. The sense in which this differential operator commutes with the representation $\rho$ is expressed in the following:

LEMMA 1. For any function $f(z) \in \mathbb{C}$ and any transformation $T \in \mathcal{H}$

$$
\mathfrak{D}_{\rho}\left(\left.f\right|_{\rho} T\right)=\left.\left(\mathfrak{D}_{\rho} f\right)\right|_{\rho \delta} T \text {. }
$$


Proof. First recall that for any $T \in \mathcal{H}$

$$
\operatorname{Im} T z=\operatorname{Im}(a z+b) /(c z+d)=\Delta_{T}|c z+d|^{-2} \operatorname{Im} z ;
$$

hence putting $q(z)=k / 2 i y, y=\operatorname{Im} z$, it follows that

$$
\begin{aligned}
q(T z) & =\frac{k}{2 i y \Delta_{T}}|c z+d|^{2} \\
& =\frac{k}{2 i y \Delta_{T}}\left\{(c z+d)^{2}-2 i c y(c z+d)\right\} \\
& =\delta(T, z) q(z)-\frac{k c}{\Delta_{T}}(c z+d) .
\end{aligned}
$$

Then for any $f \in \mathbb{C}$ and $T \in \mathcal{H}$, putting $w=T z$,

$$
\begin{aligned}
\mathfrak{D}_{\rho}\left(\left.f\right|_{\rho} T\right) & =\left(\frac{\partial}{\partial z}+q(z)\right)\left(\rho(T, z)^{-1} f(T z)\right) \\
& =\rho(T, z)^{-1} \delta(T, z)^{-1} \cdot\left\{\frac{\partial f(w)}{\partial w}+\delta(T, z) q(z) f(w)-\frac{k c}{\Delta_{T}}(c z+d) f(w)\right\} \\
& =\rho(T, z)^{-1} \delta(T, z)^{-1} \cdot\left\{\frac{\partial f(w)}{\partial w}+q(w) f(w)\right\} \\
& =\left.\left(\mathfrak{D}_{\rho} f\right)\right|_{\rho \delta} T .
\end{aligned}
$$

Defining

$$
\mathfrak{D}_{\rho}^{r}=\mathfrak{D}_{\rho \delta^{r-1}} \cdots \mathfrak{D}_{\rho \delta} \cdot \mathfrak{D}_{\rho},
$$

a repeated application of (4) shows that

$$
\mathfrak{D}_{\rho}^{r}\left(\left.f\right|_{\rho} T\right)=\left.\left(\mathfrak{D}_{\rho}^{r} f\right)\right|_{\rho \delta^{r}} T .
$$

Thus Lemma 1 also provides a family of higher order linear differential operators which commute with the representation $\rho$ in the sense of equation (6). It should perhaps be noted here that these are the only differential operators with such properties, up to constant factors; the proof of this assertion, being trivial and irrelevant, will not be given here. There is however some use for an explicit formula for the iterated operators defined by (5).

Lемма 2. If $k$ is the degree of the multiplier $\rho$ then

$$
\mathfrak{D}_{\rho}^{r}=\sum_{s=0}^{r} C_{s}^{r} \frac{(k-1)(k) \cdots(k-1+r)}{(k-1)(k) \cdots(k-1+s)}(2 i y)^{s-r} \frac{\partial^{s}}{\partial z^{s}},
$$

where $C_{s}^{\top}$ are the binomial coefficients and 


$$
\frac{\partial^{s}}{\partial z^{8}}=\frac{\partial}{\partial z} \cdots \frac{\partial}{\partial z} .
$$

Proof. The proof is of course by induction on $r$. If $r=1$ equation (7) reduces to equation (3); if then equation (7) holds for some $r \geqq 1$ it follows that

$$
\begin{aligned}
\mathfrak{D}_{\rho}^{r+1}= & \mathfrak{D}_{\rho \delta^{r}} \mathfrak{D}_{\rho}^{r} \\
= & \left(\frac{\partial}{\partial z}=\frac{k+2 r}{2 i y}\right)\left(\sum_{s=0}^{r} C_{s}^{r} \frac{(k-1) \cdots(k-1+r)}{(k-1) \cdots(k-1+s)}(2 i y)^{s-r} \frac{\partial^{s}}{\partial z^{s}}\right) \\
= & \sum_{s=0}^{r} C_{s}^{r} \frac{(k-1) \cdots(k-1+r)}{(k-1) \cdots(k-1+s)} \\
& \cdot\left\{(2 i y)^{s-r} \frac{\partial^{s+1}}{\partial z^{s+1}}+(s-r)(2 i y)^{s-r-1} \frac{\partial^{s}}{\partial z^{s}}+(k+2 r)(2 i y)^{s-r-1} \frac{\partial^{s}}{\partial z^{s}}\right\} \\
= & \frac{\partial^{r+1}}{\partial z^{r+1}}+\frac{(k-1) \cdots(k+r)}{(k-1)}(2 i y)^{-r-1}+\sum_{s=1}^{r} \frac{(k-1) \cdots(k-1+r)}{(k-1) \cdots(k-1+s)} \\
= & \sum_{s=0}^{r+1} C_{s}^{r+1} \frac{(k-1) \cdots(k+r)}{(k-1) \cdots(k-1+s)}(2 i y)^{s-r-1} \frac{\partial^{s}}{\partial z^{s}},
\end{aligned}
$$

since

$$
\begin{aligned}
& C_{s-1}^{r}(k-1+s)+C_{s}^{r}(k+r+s) \\
& \quad=(k+r) \cdot\left[C_{s}^{r}+C_{s-1}^{r}\right]+\left[s C_{s}^{r}-(r+1-s) C_{s-1}^{r}\right]=(k+r) C_{s}^{r+1} .
\end{aligned}
$$

This is just equation ( 7$)$ for the case $r+1$, thus completing the induction argument.

The vector space $\mathfrak{A}$ of functions holomorphic on $\mathbb{D}$ is a subspace of $\mathfrak{C}$ which is stable under the representation $\rho$ of $\mathscr{H}$ on $\mathfrak{E}$; hence the restriction of $\rho$ to the subspace $\mathfrak{A}$ is another linear representation of $\mathfrak{H}$, which will still be denoted by $\rho$. The linear differential operators $\mathfrak{D}_{\rho}^{r}$ of course commute with the representation $\rho$ on $\mathfrak{A}$ in the sense of equation (6), provided only that they preserve the space $\mathfrak{A}$; but it is clear from (7) that $\mathfrak{D}_{\rho}^{r} \mathfrak{A} \subset \mathfrak{A}$ if and only if $(k-1)(k) \cdots(k-1+r) /(k-1)(k) \cdots(k-1+s)=0$ for $0 \leqq s<r$, that is, if and only if $r=1-k$. Thus for the study of the space $\mathfrak{A}$ by these means one is restricted to considering multipliers $\rho$ of degree $k \leqq 0$ and the associated differential operators $\mathfrak{D}_{\rho}^{r}$ with $r=1-k$; note that in this case

$$
\mathfrak{D}_{\rho}^{1-k}=\frac{\partial^{1-k}}{\partial z^{1-k}} .
$$


2. Exact cohomology sequences associated to the differential operators. Let $\mathcal{G}$ be a properly discontinuous subgroup of $\mathfrak{T C}$ possessing a fundamental domain which can be compactified by adding a finite number of parabolic vertices [8]. The present discussion will be devoted principally to those groups $\mathcal{G}$ which have at least one parabolic vertex; the simplifications arising when there are no parabolic vertices will merely be noted in passing. In particular it will be assumed that the point at infinity is a parabolic vertex of $\mathcal{G}$, or equivalently that $\mathcal{G}$ contains translations.

If $\lambda(T)$ is an $n$-dimensional complex linear representation of the group $\mathcal{G}$, that is, a homomorphism of $\mathcal{G}$ into the group $\operatorname{GL}(n, C)$, and $\rho(T, z)$ is the scalar multiplier defined by (1), then the matrix functions $\mu(T, z)=\rho(T, z) \lambda(T)$ satisfy the functional equations $\mu\left(T_{1} T_{2}, z\right)=\mu\left(T_{1}, T_{2} z\right) \mu\left(T_{2}, z\right)$ for all $T_{1}, T_{2} \in \mathcal{G}$ and hence define a matrix factor of automorphy for the group $\mathcal{G}$. Let $\mathfrak{E}_{n}=\mathfrak{C}+\cdots+\mathfrak{C}$, an element $F=F(z) \in \mathfrak{S}_{n}$ being considered as a column vector of $n$ functions $f_{i}(z) \in \mathcal{C}$. The mapping $\left.(F, T) \rightarrow F\right|_{\mu} T$ of $\mathfrak{夭}_{n} \times \mathcal{G}$ into $\mathcal{G}$ defined by

$$
\left(F \mid{ }_{\mu} T\right)(z)=\mu(T, z)^{-1} F(T z)
$$

is then a representation of $\mathcal{G}$ as a group of linear transformations on the complex vector space $\mathfrak{E}_{n}$; this representation will be denoted by $\mu$ as well.

Now select a subset $\beta$ of the group $\mathfrak{F}$ such that:

(i) $\mathrm{S \subset GP \subset P \text {; }}$

(ii) for each parabolic fixed point $z_{0}$ of $\mathcal{G}$ there are transformations $P \in \odot$, and at most finitely many, such that $P \infty=z_{0}$; and

(iii) for each transformation $P \in \mathcal{P}$ the point $P \infty$ is a parabolic fixed point of $\mathrm{g}$.

This set will be assumed fixed in what follows. Then for any real number $\epsilon \geqq 0$ let $\mathbb{C} \epsilon$ be the subspace of $\mathbb{C}$ consisting of those $C^{\infty}$ functions $f(z)$ on $D$ such that:

for each transformation $P \in \mathcal{P}$ and for each real number $c>\epsilon$ the func-

tion $g(z)=\left(\left.f\right|_{\rho} P\right)(z)$ satisfies the condition that $e^{-c y}|g(z)| \rightarrow 0$ uniformly as $y \rightarrow+\infty$ in any strip $a<x<b$ of finite width;

and let $\mathfrak{S}_{n}^{\epsilon}=\mathfrak{S}_{\epsilon}+\cdots+\mathfrak{S}_{\epsilon} \subset \mathfrak{C}_{n}$. It follows immediately from (10)(i) that $\left.F\right|_{\mu} T \in \mathfrak{G}_{n}^{\epsilon}$ whenever $F \in \mathfrak{S}_{n}^{\epsilon}$ and $T \in \mathcal{G}$; hence $\mu$ also determines a linear representation of the group $\mathcal{G}$ on each subspace $\mathfrak{F}_{n}^{\epsilon} \subset \mathcal{S}_{n}$. If $\mathcal{G}$ has no parabolic vertices this entire construction is of course unnecessary.

To introduce cohomological machinery recall, from [7] for instance, that a homogeneous $p$-cochain of $\mathcal{G}$ with coefficients in $\mathfrak{C}_{n}$ associated to the representation $\mu$ of $\mathcal{G}$ as a group of linear transformations of $\mathbb{S}_{n}$ is a function $F\left(T_{0}, T_{1}, \cdots, T_{p}\right)$ of $p+1$ variables $T_{i} \in \mathcal{G}$ with values in $\mathfrak{S}_{n}$ such that

$$
F\left(T_{0} T, T_{1} T, \cdots, T_{p} T\right)=\left.F\left(T_{0}, T_{1}, \cdots, T_{p}\right)\right|_{\mu} T
$$


for all $T, T_{i} \in \mathcal{G}$; the set of all such $p$-cochains forms a complex vector space $C_{\mu}^{p}\left(\mathcal{G}, \mathbb{S}_{n}\right)$ in the obvious manner. The coboundary operator $\delta: C_{\mu}^{p}\left(\mathcal{G}, \mathfrak{E}_{n}\right)$ $\rightarrow C_{\mu}^{p+1}\left(\mathcal{G}, \mathbb{E}_{n}\right)$ is the linear mapping which associates to any cochain $F\left(T_{0}, \cdots, T_{p}\right) \in C_{\mu}^{p}\left(\mathcal{G}, \mathfrak{S}_{n}\right)$ the cochain

$$
(\delta F)\left(T_{0}, \cdots, T_{p+1}\right)=\sum_{i=0}^{p+1}(-1)^{i} F\left(T_{0}, \cdots, T_{i-1}, T_{i+1}, \cdots, T_{p+1}\right)
$$

in $C_{\mu}^{p+1}\left(\mathcal{G}, \mathfrak{S}_{n}\right)$. The kernel of this mapping is the subspace $Z_{\mu}^{p}\left(\mathcal{G}, \mathfrak{S}_{n}\right) \subset C_{\mu}^{p}\left(\mathcal{G}, \mathfrak{S}_{n}\right)$ of cocycles, and the cohomology groups are the quotient spaces

$$
H_{\mu}^{p}\left(\mathcal{G}, \mathfrak{\mho}_{n}\right)=Z_{\mu}^{p}\left(\mathcal{G}, \mathfrak{\mho}_{n}\right) / \delta C_{\mu}^{p-1}\left(\mathcal{G}, \mathfrak{\mho}_{n}\right) .
$$

There are in addition cohomology groups $H_{\mu}^{p}\left(\mathcal{G}, \mathfrak{B}_{n}\right)$ associated to any subspaces $\mathfrak{B}_{n} \subset \mathfrak{C}_{n}$ which are stable under the representation $\mu$, as for example the cohomology groups $H_{\mu}^{p}\left(\mathcal{G}, \mathfrak{S}_{n}^{\epsilon}\right)$. More important for the present purposes are the cohomology groups $H_{\mu}^{p}\left(\mathcal{G}, \mathfrak{A}_{n}\right)$ and $H_{\mu}^{p}\left(\mathfrak{G}, \mathfrak{A}_{n}^{\epsilon}\right)$, where $\mathfrak{A}_{n}=\mathfrak{A}+\cdots+\mathfrak{A}$ $\subset \mathfrak{S}_{n}$ is the subspace of holomorphic functions and $\mathfrak{A}_{n}^{\epsilon}=\mathfrak{A}_{n} \cap \mathfrak{F}_{n}^{\epsilon}$. A useful relationship among these groups follows directly from the results of $\S 1$, after the preliminary:

Lемма 3. If the multiplier $\rho$ is of degree $k \leqq 0$ then for any $\epsilon \geqq 0$ the following is an exact sequence of complex vector spaces and linear maps

$$
0 \rightarrow \mathfrak{B}(-k) \stackrel{i}{\rightarrow} \mathfrak{A} \epsilon \stackrel{\mathfrak{D}_{\rho}^{1-k}}{\longrightarrow} \mathfrak{A} \epsilon \rightarrow 0
$$

where $i$ is the inclusion map and $\mathfrak{B}(-k)$ is the space of complex polynomials of degree $\leqq-k$.

Proof. It follows immediately from $(8)$ and $(11)$ that $\mathfrak{B}(-k)$ is precisely the kernel of the linear map $\mathfrak{D}_{\rho}^{1-\boldsymbol{z}}$. If $f(z) \in \mathfrak{A} \epsilon, P \in \mathcal{P}$, and $g=\left.f\right|_{\rho} P$, then by (6)

$$
\begin{aligned}
\left.\left(\mathfrak{D}_{\rho}^{1-k} f\right)\right|_{\rho} P & =\delta^{1-k}(P, z) \frac{d^{1-k} g(z)}{d z^{1-k}} \\
& =\delta^{1-k}(P, z) \frac{(1-k) !}{2 \pi i} \oint(\zeta-z)^{k-2} g(\zeta) d \zeta ;
\end{aligned}
$$

hence if $g(z)$ satisfies the growth condition of (11) so does $\left.\left(\mathfrak{D}_{\rho}^{1-k} f\right)\right|_{\rho} P$, and therefore $\mathfrak{D}_{\rho}^{1-k} \mathfrak{Q} \mathfrak{C}^{\epsilon} \subset \mathfrak{A} \epsilon$. On the other hand if $f(z) \in \mathfrak{A}^{\epsilon}$ and $P \in \mathcal{P}$ then the iterated indefinite integral

$$
g_{P}(z)=\frac{1}{(-k) !} \int_{z_{0}}^{z}(z-\zeta)^{-k}\left(\left.f\right|_{\rho \delta^{1-k}} P\right)(\zeta) d \zeta
$$

represents a function in $\mathfrak{A}$ which is readily seen to satisfy the growth condition of (11) and the differential equation $\mathfrak{D}_{\rho}^{1-\boldsymbol{k}} g_{P}=\left.f\right|_{\rho \delta^{1-k}} P$. In particular 
$g(z)=g_{I}(z)$, where $I$ is the identity element of the group $G$, satisfies $\mathfrak{D}_{\rho}^{1-\boldsymbol{z}} g=f$; and since by (6) $\mathfrak{D}_{\rho}^{1-k}\left(\left.g\right|_{\rho} P-g_{P}\right)=0$, then $\left.g\right|_{\rho} P-g_{P}$ is a polynomial, so that $\left.g\right|_{\rho} P$ satisfies the same growth condition as does $g_{P}$ and hence $g \in \mathfrak{A} \epsilon$. Therefore $\mathfrak{U}^{\epsilon} \subset \mathfrak{D}_{\rho}^{1-k} \mathfrak{Q} \mathfrak{\epsilon}$, which completes the proof.

THEOREM 1. If the multiplier $\rho$ is of degree $k \leqq 0$ then for any $\epsilon \geqq 0$ the following is an exact sequence of complex vector spaces and linear maps:

$$
\begin{gathered}
0 \rightarrow H_{\mu}^{0}\left(\mathcal{G}, \mathfrak{B}(-k)_{n}\right) \stackrel{i}{\rightarrow} H_{\mu}^{0}\left(\mathcal{G}, \mathfrak{A}_{n}^{\epsilon}\right) \stackrel{\mathfrak{D}_{\rho}^{1-k}}{\rightarrow} H_{\mu \delta^{1-k}}^{0}\left(\mathcal{G}, \mathfrak{A}_{n}^{\epsilon}\right) \\
\stackrel{\delta}{\rightarrow} H_{\mu}^{1}\left(\mathcal{G}, \mathfrak{B}(-k)_{n}\right) \stackrel{i}{\rightarrow} H_{\mu}^{1}\left(\mathcal{G}, \mathfrak{A}_{n}^{\epsilon}\right) \stackrel{\mathfrak{D}_{\rho}^{1-k}}{\longrightarrow} \cdots \\
\ldots \stackrel{\delta}{\rightarrow} H_{\mu}^{p}\left(\mathcal{G}, \mathfrak{B}(-k)_{n}\right) \stackrel{i}{\rightarrow} H_{\mu}^{p}\left(\mathcal{G}, \mathfrak{A}_{n}^{\epsilon}\right) \stackrel{\mathfrak{D}_{\rho}^{1-k}}{\longrightarrow} H_{\mu \delta^{1-k}}^{p}\left(\mathcal{G}, \mathfrak{A}_{n}^{\epsilon}\right) \stackrel{\delta}{\rightarrow} \cdots
\end{gathered}
$$

Proof. The direct sum of $n$ copies of the exact sequence (15) of Lemma 3 gives an exact sequence

$$
0 \rightarrow \mathfrak{B}(-k)_{n} \stackrel{i}{\rightarrow} \mathfrak{A}_{n}^{\epsilon} \stackrel{\mathfrak{D}_{\rho}^{1-k}}{\longrightarrow} \mathfrak{A}_{n}^{\epsilon} \rightarrow 0 ;
$$

since $\lambda(T)$ are constants it follows from (6) that $\mathfrak{D}_{\rho}^{1-k}\left(\left.F\right|_{\mu} T\right)=\left.\left(\mathfrak{D}_{\rho}^{1-k} F\right)\right|_{\mu \delta^{1-k}} T$ for all $F \in \mathfrak{A}_{n}^{\epsilon}$ and $T \in \mathcal{G}$, hence that (17) determines an exact cochain sequence

$$
0 \rightarrow C_{\mu}^{p}\left(\mathcal{S}, \mathfrak{B}(-k)_{n}\right) \stackrel{i}{\rightarrow} C_{\mu}^{p}\left(\mathcal{G}, \mathfrak{A}_{n}^{\epsilon}\right) \stackrel{\mathfrak{I}_{\rho}^{1-k}}{\longrightarrow} C_{\mu \delta^{1-k}}^{p}\left(\mathcal{S}, \mathfrak{A}_{n}^{\epsilon}\right) \rightarrow 0
$$

which clearly commutes with the coboundary operator. Any such cochain sequence is well known to determine an associated exact cohomology sequence of the form (16) [4].

3. The zero-dimensional analytic cohomology groups. The zero-dimensional cohomology groups are quite easy to interpret, since first of all $H_{\mu}^{0}\left(\mathcal{G}, \mathfrak{A}_{n}^{\epsilon}\right)$ $=Z_{\mu}^{0}\left(\mathcal{G}, \mathfrak{A}_{n}^{\epsilon}\right)$. A zero-cochain $F\left(T_{0}\right) \in C_{\mu}^{0}\left(\mathcal{G}, \mathfrak{A}_{n}^{\epsilon}\right)$ is determined uniquely by its inhomogeneous form $F=F(I) \in \mathfrak{A}_{n}^{e}$, where $I$ is the identity element of the group $\mathcal{G}$; and conversely any element $F \in \mathfrak{A}_{n}^{\epsilon}$ is the inhomogeneous form of the zero-cochain $F\left(T_{0}\right)=\left.F\right|_{\mu} T_{0}$. Identifying the zero-cochains with their inhomogeneous forms, the zero-cocycles correspond to those elements $F \in \mathfrak{A}_{n}^{\epsilon}$ such that $\left.F\right|_{\mu} T-F=0$ for all $T \in \mathcal{G}$; that is,

$$
Z_{\mu}^{0}\left(\mathcal{G}, \mathfrak{A}_{n}^{\epsilon}\right)=\left\{F \in \mathfrak{A}_{n}^{\epsilon} \mid F(T z)=\mu(T, z) F(z), \text { all } T \in \mathcal{G}\right\} .
$$

Thus $H_{\mu}^{0}\left(\mathcal{G}, \mathfrak{A}_{n}^{\epsilon}\right)$ can be interpreted as a space of automorphic forms for the group $\mathcal{G}$ associated to the factor of automorphy $\mu$; no further comment is necessary for groups without parabolic vertices, but for groups with parabolic vertices a slightly more detailed investigation of the behavior of these forms 
at the parabolic vertices is required to identify them with the automorphic forms as customarily defined [8].

For the purpose suppose that the kernel of $\lambda$ is a subgroup $g_{\lambda}$ of finite index in $\mathcal{G}_{\text {; then }} \mathcal{G}_{\lambda}$ and $\mathcal{G}_{\text {have }}$ the same parabolic fixed points. If $F \in Z_{\mu}^{0}\left(\mathcal{G}, \mathfrak{A}_{n}\right)$ each component $f$ of $F$ satisfies $\left.f\right|_{\rho} T=f$ for all $T \in \mathcal{S}_{\lambda}$, that is, $f \in Z_{\rho}^{0}\left(\mathcal{G}_{\lambda}, \mathfrak{U}\right)$; if further $z_{0}$ is any parabolic fixed point for $\mathcal{G}$ and $P \in \mathcal{H}$ is a transformation such that $P \infty=z_{0}$ then $\left.\left(\left.f\right|_{\rho} P\right)\right|_{\rho}\left(P^{-1} T P\right)=\left.f\right|_{\rho} P$ for all $T \in \mathcal{G}_{\lambda}$, that is $\left.f\right|_{\rho} P$ $\in Z_{\rho}^{0}\left(P^{-1} \mathcal{G}_{\lambda} P, \mathfrak{A}\right)$. Letting $T_{P}: z \rightarrow z+b, b>0$, generate the translation subgroup of $P^{-1} G_{\lambda} P$, it follows that $\left(\left.f\right|_{\rho} P\right)(z+b)=\left(\left.f\right|_{\rho} P\right)(z)$, hence that the function $\left.f\right|_{\rho} P$ has a Fourier expansion

$$
\left(\left.f\right|_{\rho} P\right)(z)=\sum_{r=-\infty}^{\infty} a_{r} \exp (2 \pi i r z / b)
$$

The function $f$ is said to be holomorphic at $z_{0}$ if $a_{r}=0$ for all $r<0$, and to vanish at $z_{0}$ if moreover $a_{0}=0$; note that this is independent of the choice of transformation $P$. The set of all functions $F \in Z_{\mu}^{0}\left(\mathcal{G}, \mathfrak{A}_{n}\right)$ each component of which is holomorphic at all parabolic vertices of $G$ form a complex vector space $\Gamma_{\mu}(\mathcal{G})$, the space of automorphic forms for the group $G$ associated to the factor of automorphy $\mu$; the subspace $\Gamma_{\mu}^{0}(\mathcal{G})$ of those automorphic forms which moreover vanish at all parabolic vertices of $g$ is the space of cusp forms (or Spitzenformen) for the group $\mathcal{G}$ associated to the factor of automorphy $\mu$.

THEOREM 2. If the kernel of $\lambda$ is of finite index in $\mathcal{G}$ there is an $\epsilon_{0}>0$ such that

$$
H_{\mu}^{0}\left(\mathcal{S}, \mathfrak{A}_{n}^{\epsilon}\right)=\Gamma_{\mu}(\mathcal{S})
$$

for all $\epsilon<\epsilon_{0}$.

Proof If $F \in \Gamma_{\mu}(\mathcal{G}), f$ is a component of $F$, and $P \in \mathcal{P}$, then by (10)(iii) $P \infty=z_{0}$ is a parabolic vertex of $\mathcal{G}$ so that $\left(\left.f\right|_{\rho} P\right)(z)=g(z)$ will have a Fourier expansion (20) with $a_{r}=0$ for all $r<0$; consequently $g(z) \rightarrow a_{0}$ uniformly as $y \rightarrow \infty$, so that $e^{-c y}|g(z)| \rightarrow 0$ uniformly as $y \rightarrow \infty$ for any $c>0$, and $f(z) \in \mathfrak{A}^{0}$. Thus $\Gamma_{\mu}(\mathcal{G}) \subset H_{\mu}^{0}\left(\mathcal{G}, \mathfrak{A}_{n}^{0}\right) \subset H_{\mu}^{\epsilon}\left(\mathcal{G}, \mathfrak{A}_{n}^{0}\right)$ for all $\epsilon>0$. Conversely if $F \in H_{\mu}^{0}\left(\mathcal{G}, \mathfrak{A}_{n}^{\epsilon}\right)$, $f$ is a component of $F$, and $z_{0}$ is a parabolic vertex of $G$, then by (10)(ii) there is a transformation $P \in \mathcal{P}$ such that $P \infty=z_{0}$, hence $\left(\left.f\right|_{\rho} P\right)(z)=g(z)$ will have a Fourier expansion (20). If $e^{-c y}|g(z)| \rightarrow 0$ uniformly as $y \rightarrow \infty$ for some $c \leqq 2 \pi / b$ then for all $r<0$

$$
\left|a_{r}\right|=\frac{1}{b} \cdot\left|\int_{0}^{b} \exp (-2 \pi i r z / b) f(z) d z\right| \leqq \frac{1}{b} \int_{0}^{b} e^{-c y}|f(z)| d x
$$

so that $a_{r}=0$. Since there are at most finitely many parabolic vertices inequivalent under $\mathcal{G}_{\lambda}$, then setting $\epsilon_{0}=\min _{b}(2 \pi / b)>0$ it follows that for $\epsilon<\epsilon_{0}$, $F(z)$ is holomorphic at all the parabolic vertices of $\mathcal{G}$. Thus for $\epsilon<\epsilon_{0}, H_{\mu}^{0}\left(\mathcal{G}, \mathfrak{A}_{n}^{\epsilon}\right)$ $\subset \Gamma_{\mu}(\mathcal{G})$, which completes the proof. 
4. The one-dimensional analytic cohomology groups. Turning next to the one-dimensional cohomology groups, a one-cochain $F\left(T_{0}, T_{1}\right) \in C_{\mu}^{1}\left(\mathcal{G}, \mathfrak{A}_{n}^{\epsilon}\right)$ is determined uniquely by its inhomogeneous form $F(T)=F(I, T)$, where $I$ is the identity element of the group $\mathcal{G}$; and conversely any function $F(T)$ from $\mathcal{G}$ to $\mathfrak{A}_{n}^{\epsilon}$ is the inhomogeneous form of a one-cochain $F\left(T_{0}, T_{1}\right)=F\left(T_{1} T_{0}^{-1}\right) \mid{ }_{\mu} T_{0}$. Identifying the functions $F(T)$ with the one-cochains it follows from (13) that the space $Z_{\mu}^{1}\left(\mathcal{G}, \mathfrak{A}_{n}^{\bullet}\right)$ of one-cocycles consists of those functions $F(T)$ such that

$$
F\left(T_{1} T_{2}\right)=\left.F\left(T_{1}\right)\right|_{\mu} T_{2}+F\left(T_{2}\right)
$$

for all $T_{1}, T_{2} \in \mathcal{G}$, and that the space $\delta C_{\mu}^{0}\left(\mathcal{S}, \mathfrak{A}_{n}^{\epsilon}\right)$ of one-coboundaries consists of those functions $F(T)$ of the form

$$
F(T)=\left.G\right|_{\mu} T-G
$$

for some $G \in \mathfrak{A}_{n}^{\epsilon}$. The group $H_{\mu}^{1}\left(\mathcal{G}, \mathfrak{A}_{n}^{\epsilon}\right)=Z_{\mu}^{1}\left(\mathcal{G}, \mathfrak{A}_{n}^{\epsilon}\right) / \delta C_{\mu}^{1}\left(\mathcal{G}, \mathfrak{A}_{n}^{\epsilon}\right)$ is then the quotient of the space of functions $F(T)$ from $\mathcal{G}$ to $\mathfrak{A}_{n}^{e}$ satisfying (22) modulo the subspace of functions of the form (23).

Lemma 4. If $F(z) \in \mathfrak{Y}_{n}^{0}$ and $\lambda \in G L(n, C)$ is an element of finite order then for any $\delta>0, \epsilon>0$ there exists a vector-valued function $G(z)$ holomorphic in the half-plane $y>\delta$ such that:

$$
\lambda^{-1} G(z+1)-G(z)=F(z) ;
$$

and

for any $c>\epsilon$ and any component $g(z)$ of $G(z), e^{-c y}|g(z)| \rightarrow 0$ uniformly as $y \rightarrow+\infty$ in any strip of finite width.

Proof. It follows by induction from (24) that for any integer $a \geqq 1$, $\lambda^{-a} G(z+a)-G(z)=F_{a}(z)$ where

$$
F_{a}(z)=\sum_{r=0}^{a-1} \lambda^{-r} F(z+r)
$$

and if $a$ is a multiple of the order of $\lambda$ then $\lambda^{-a}=1$. The first step in the proof is the construction of a function $G_{a}(z)$ holomorphic in $y>\delta$ which satisfies (25) and

$$
G_{a}(z+a)-G_{a}(z)=F_{a}(z),
$$

where the integer $a$ is a multiple of the order of $\lambda$ and $a \geqq 2 \pi / \epsilon$; it of course suffices to consider a single component $f(z)$ of the vector-valued function $F_{a}(z)$. Following the approach used in [1] let

$$
k(z, t)=a^{-1}(1-\exp \{2 \pi i(z-t) / a\})^{-1}
$$

where $z=x+i y, t=\sigma+i \tau$. For each fixed $z$ the function $k(z, t)$ is meromorphic 
in $t$, the only singularities being simple poles at the points $t=z, z \pm a$, $z \pm 2 a, \cdots \quad$ with residues $1 / 2 \pi i ; k(z+a, t)=k(z, t)$; and $|k(z, t)|$ $<(\exp \{2 \pi(y-\tau) / a\}) / a$ if $y-\tau>a$. Since $f(z) \in \mathfrak{A}^{0}$ it follows that the integral $h(z)=\int_{i \delta}^{i \infty} k(z, t) f(t) d t$ extended over the line segment $\sigma=0, \delta<\tau<\infty$ converges to a function holomorphic in each strip $m a<x<(m+1) a$ for $m=0, \pm 1, \pm 2, \cdots$, and that $h(z+a)=h(z)$. Modifying the path of integration by making indentations to the left or right respectively shows that the function $h(z)$ has analytic continuations $h^{+}(z), h^{-}(z)$ from the right and left respectively across the lines $x=m a$, and as in [1] $h^{-}(z)-h^{+}(z)=f(z)$ along the line $x=0$. Considering the function $h(z)$ in the single strip $0<x<a$ the analytic continuations across the two sides of the strip are such that $h(z+1)$ $-h(z)=h^{-}(z)-h^{+}(z)=f(z)$; therefore this segment of the function can be extended to a function $g_{a}(z)$ holomorphic in the half-plane $y>\delta$ such that $g_{a}(z+1)-g_{a}(z)=f(z)$. If $(x-\sigma) / a$ is not an integer then $|k(z, t)|$ $\leqq a^{-1}|\sin 2 \pi(x-\sigma) / a|^{-1} \exp \{2 \pi(y-\tau) / a\}$ for all $y$; hence in any strip $\sigma+\eta<x<\sigma+a-\eta$ for any $\eta>0$

$$
\begin{aligned}
& e^{-c y}\left|g_{a}(z)\right| \leqq e^{-c y}\left|\int_{i \delta}^{\sigma+i \delta} k(z, t) f(t) d t\right|+e^{-c y}\left|\int_{\sigma+i \delta}^{\sigma+i \infty} k(z, t) f(t) d t\right| \\
& \leqq C e^{-c y}+\frac{1}{a}\left|\sin \frac{2 \pi \eta}{a}\right|^{-1} \exp \{-(c-2 \pi / a) y\} \int_{\delta}^{\infty} \exp \{-2 \pi \tau / a\}|f(\sigma+i \tau)| d \tau
\end{aligned}
$$

so that $e^{-c y}\left|g_{a}(z)\right| \rightarrow 0$ uniformly as $y \rightarrow \infty$ whenever $c>\epsilon \geqq 2 \pi / a$.

Now in terms of the auxiliary function $G_{a}(z)$ define another function by setting

$$
G(z)=\frac{1}{a} \sum_{r=0}^{a-1}\left(\lambda^{-r} G_{a}(z+r)-F_{r}(z)\right),
$$

where $F_{r}(z)$ is given by (26) for $r>0$ and $F_{0}(z)=0$. If $G_{a}(z)$ satisfies (25) then so does $G(z)$; and

$$
\begin{aligned}
\lambda^{-1} G(z+1) & =\frac{1}{a} \sum_{r=0}^{a-1}\left(\lambda^{-r-1} G_{a}(z+r+1)-F_{r+1}(z)+F(z)\right) \\
& =F(z)+G(z)+\frac{1}{a}\left(G_{a}(z+a)-G_{a}(z)-F_{a}(z)\right) \\
& =F(z)+G(z) .
\end{aligned}
$$

This therefore completes the proof.

As a convenient abbreviation an open subset $\mathcal{U} \subset D$ will be called an open neighborhood of $\infty$ if $\mathcal{u}$ contains a half-plane $\operatorname{Im} z>y_{0}$; and $\mathcal{u}$ will be called an open neighborhood of a finite parabolic vertex $z_{0}$ if $\mathcal{u}$ contains an open circular disc in $D$ tangent to the real axis at $z_{0}$, or equivalently, if $P^{-1} \mathcal{U}$ is an open neighborhood of $\infty$ for some $P \in \mathcal{P}$ such that $P \infty=z_{0}$. The set of those 
$C^{\infty}$ vector-valued functions in $D$ which are holomorphic in an open neighborhood of each parabolic vertex of $\mathcal{G}$ is a subspace $\tilde{\mathbb{E}}_{n} \subset \mathscr{E}_{n}$ stable under the representation $\mu$; hence $\tilde{\mathfrak{E}}_{n}^{\epsilon}=\widetilde{\mathfrak{E}}_{n} \cap \mathfrak{E}_{n}^{\epsilon}$ is also stable so that the cohomology groups $H_{\mu}^{1}\left(\mathcal{G}, \tilde{\mathfrak{C}}_{n}^{\epsilon}\right)$ are well defined.

Lemma 5. If the kernel of $\lambda$ is of finite index in 9 then for any $\epsilon>0$ the homomorphism $H_{\mu}^{1}\left(\mathcal{G}, \widetilde{\mathfrak{G}}_{n}^{0}\right) \rightarrow H_{\mu}^{1}\left(\mathcal{G}, \widetilde{\mathfrak{G}}_{n}^{\epsilon}\right)$ induced by the inclusion map $\tilde{\mathfrak{G}}_{n}^{0} \rightarrow \widetilde{\mathfrak{G}}_{n}^{\epsilon}$ is the zero homomorphism.

Proof. What is to be proved is that any one-cocycle $F(T) \in Z_{\mu}^{1}\left(\mathcal{G}, \widetilde{\mathfrak{C}}_{n}^{0}\right)$ is the coboundary of a zero-cochain $G \in C_{\mu}^{0}\left(\mathcal{G}, \widetilde{\widetilde{G}}_{n}^{e}\right)$. For this purpose first select a pair of open neighborhoods $\mathfrak{U}_{i}, \mathcal{V}_{i}$ of each parabolic vertex of $\mathcal{G}$ such that the sets $\vartheta_{i}$ are pairwise disjoint, the point set closure of $\mathcal{U}_{i}$ is contained in $\mathcal{V}_{i}$, and the sets $\mathcal{U}=\cup \mathcal{U}_{i}, \mathcal{V}=\bigcup \mathcal{V}_{i}$ are invariant under the group $\mathcal{G}$ and contain no fixed points of $\mathcal{G}$; and select a $C^{\infty}$ function $\eta=\eta(z)$ in $D$ invariant under $\mathcal{G}$ and such that $0 \leqq \eta(z) \leqq 1, \eta(z)=0$ for $z \in D-\mathcal{V}, \eta(z)=1$ for $z \in \mathcal{U}$. Now for each $\mathfrak{u}_{i}$ there is a transformation $P_{i} \in \mathcal{P}$ such that $P_{i}^{-1} \mathfrak{u}_{i}$ is a halfplane $\operatorname{Im} z>y_{i}$; and if $T_{i}$ generates the cyclic subgroup of those parabolic transformations in $\mathcal{G}$ preserving the set $\mathcal{U}_{i}$ then $P_{i}^{-1} T_{i} P_{i}$ is a translation $z \rightarrow z+b_{i}$. By Lemma 4 there is a holomorphic function $G_{i}^{\prime \prime}(z)$ in $P_{i}^{-1} \mathfrak{u}_{i}$ satisfying (25) and such that $\lambda\left(T_{i}\right)^{-1} G_{i}^{\prime \prime}\left(z+b_{i}\right)-G_{i}^{\prime \prime}(z)=F\left(T_{i}\right) \mid{ }_{\rho} P_{i}$, since $\lambda\left(T_{i}\right)$ is a matrix of finite order as a consequence of the hypothesis; then $G_{i}^{\prime}$ $=\left.G_{i}^{\prime \prime}\right|_{\rho} P_{i}^{-1}$ is holomorphic in $\mathcal{U}_{i},\left.G_{i}\right|_{\rho} P_{i}$ satisfies (25), and $\left.G_{i}^{\prime}\right|_{\mu} T_{i}$ $=\left.\lambda\left(T_{i}\right)^{-1} G_{i}^{\prime}\right|_{\rho} T_{i}=\lambda\left(T_{i}\right)^{-1}\left(G_{i}^{\prime \prime} \mid{ }_{\rho} P_{i}^{-1} T_{i} P_{i}\right){ }_{\rho} P_{i}^{-1}=\left.\left(\lambda\left(T_{i}\right)^{-1} G_{i}^{\prime \prime}\left(z+b_{i}\right)\right)\right|_{\rho} P_{i}^{-1}$ $=G_{i}^{\prime}+F\left(T_{i}\right)$ in the set $\mathcal{U}_{i}$. Extend $G_{i}^{\prime}$ to a function $G^{\prime}$ in the entire set $\mathcal{G} \mathcal{U}_{i}$ by requiring that $\left.G^{\prime}\right|_{\mu} T-G^{\prime}=F(T)$ for all $T \in \mathcal{G}$; and proceed similarly for the other components of the set $\mathcal{U}$. It is then clear that $\eta G^{\prime} \in C_{\mu}^{0}\left(\mathcal{G}, \tilde{\mathscr{C}}_{n}^{\epsilon}\right)$ has as its coboundary the one-cocycle $\eta F(T) \in Z_{\mu}^{1}\left(\mathcal{G}, \widetilde{\mathfrak{G}}_{n}^{0}\right)$. To complete the proof it therefore suffices to prove that a one-cocycle $(1-\eta) F(T) \in Z_{\mu}^{1}\left(\mathcal{G}, \mathbb{E}_{n}\right)$ which vanishes in the open set $\mathcal{U}$ is the coboundary of a zero-cochain $G \in C_{\mu}^{0}\left(\mathcal{G}, \mathfrak{E}_{n}\right)$ which also vanishes in the set $\mathcal{u}$; but since this follows precisely as in $[9$, especially Theorem 1], the lemma is thereby demonstrated.

The preceding preparatory lemmas will now be used to establish a dual pairing $H_{\mu}^{1}\left(\mathcal{G}, \mathfrak{A}_{n}^{0}\right) \otimes \Gamma_{\hat{\mu}}^{0}(\mathcal{G}) \rightarrow C$, where $\hat{\mu}$ is the factor of automorphy defined by

$$
\hat{\mu}(T, z)=\rho(T, z)^{-1} \delta(T, z) \bar{\lambda}(T)
$$

and $\bar{\lambda}(T)$ is the complex conjugate of $\lambda(T)$. Assuming again that the kernel of $\lambda$ is of finite index in $\mathcal{G}$ there exists a positive definite Hermitian matrix $M$ such that $\lambda(T) M \bar{\lambda}(T)=M$ for all $T \in \mathcal{G}$, where ${ }^{t} \lambda(T)$ is the transpose of the matrix $\lambda(T)$. Consider elements $F(T) \in Z_{\mu}^{1}\left(\mathcal{G}, \mathfrak{A}_{n}^{0}\right)$ and $H \in \Gamma_{\hat{\mu}}^{0}(\mathcal{G})$. By Lemma 5 for any $\epsilon>0$ there exists an element $G \in \widetilde{\widetilde{G}}_{n}^{e}$ such that $F(T)=\left.G\right|_{\mu} T-G$ for all $T \in G$; then $\phi(z)=\bar{\partial} G(z)$ is a vector-valued $C^{\infty}$ differential form on $D$ of type $(0,1)$ which vanishes in an open neighborhood of each parabolic vertex of $\mathcal{S}$ and which satisfies the functional equation $\phi(T z)=\mu(T, z) \phi(z)$ 
$=\rho(T, z) \lambda(T) \phi(z)$ for all $T \in \mathcal{G}$. Associate to $H=H(z)$ the vector-valued differential form $\theta(z)=H(z) d z$; this is then a holomorphic differential form on $D$ of type $(1,0)$ which satisfies the functional equation $\theta(T z)=\rho(T, z)^{-1} \bar{\lambda}(T) \theta(z)$ for all $T \in \mathcal{G}$. It follows immediately that the expression ${ }^{t} \phi(z) \wedge M \theta(z)$ is a scalar differential form on $D$ of type $(1,1)$ which vanishes in an open neighborhood of each parabolic vertex of $\mathcal{G}$ and which is invariant under the group $\mathcal{G}$, hence that the integral

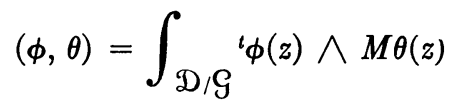

converges and is independent of the choice of fundamental domain for $\mathcal{G}$ over which the integration is extended. If $\phi, \phi^{\prime}$ are two differential forms associated to the same cocycle $F(T)$ then $\phi-\phi^{\prime}=\bar{\partial} G_{0}$ for some $G_{0} \in Z_{\mu}^{0}\left(\mathcal{G}, \tilde{\mathfrak{C}}_{n}^{\epsilon}\right)$; and as in Theorem 2 there is an $\epsilon_{0}>0$ such that for $\epsilon<\epsilon_{0}$ any function $G_{0}(z) \in Z_{\mu}^{0}\left(\mathcal{G}, \widetilde{\mathfrak{G}}_{n}^{\epsilon}\right)$ must actually be a holomorphic modular form in the usual sense near each parabolic vertex of $g$. Now the differential form ${ }^{t} G_{0}(z) M \theta(z)$, which is clearly invariant under $\mathcal{G}$, must behave like a cusp form near each parabolic vertex of $\mathcal{G}$, so that for any fundamental domain $\mathcal{F}$ with boundary $\partial \mathcal{F}$ consisting of finitely many rectifiable arcs

$$
\begin{aligned}
(\phi, \theta)-\left(\phi^{\prime}, \theta\right)=\left(\phi-\phi^{\prime}, \theta\right) & =\int_{\mathcal{F}} \bar{\partial}\left({ }^{t} G_{0}\right) \wedge M \theta=\int_{\mathcal{F}} d\left({ }^{t} G_{0} M \theta\right) \\
& =\int_{\partial \mathcal{F}}{ }^{t} G_{0} M \theta=0 .
\end{aligned}
$$

Thus for $\epsilon<\epsilon_{0}$ the inner product (29) depends only upon the cocycle $F(T)$ and not upon the choice of the associated differential form $\phi(z)$. Since moreover a coboundary $F(T)$ has an associated differential form $\phi(z) \equiv 0$ the inner product (29) actually depends only upon the cohomology class of $F(T)$. Consequently the inner product (29) defines a bilinear pairing $(F(T), H)$ of $H_{\mu}^{1}\left(\mathcal{G}, \mathfrak{A}_{n}^{0}\right) \otimes \Gamma_{\hat{\mu}}^{0}(\mathcal{G})$ into the complex numbers. As a final comment note that to calculate the inner product $(F(T), H)$ it is not necessary that the associated function $G(z)$ be holomorphic in an open neighborhood of the parabolic vertices, although that is perhaps the most convenient formulation for proving the existence of the inner product; indeed it is clearly sufficient that $G(z)$ be such that Stokes' theorem is applicable in the form $\int_{\mathscr{F}} d\left({ }^{t} G M \theta\right)=\int_{\partial F^{t}} G M \theta$.

Lемма 6. If the kernel of $\lambda$ is of finite index in 9 and if $k \leqq 0$, where $k$ is the degree of the multiplier $\rho$, then for each nontrivial $H \in \Gamma_{\hat{\mu}}^{0}(\mathcal{G})$ there exists a cohomology class $\{F(T)\} \in H_{\mu}^{1}\left(\mathcal{G}, \mathfrak{P}_{n}^{0}\right)$ such that $(F(T), H) \neq 0$.

Proof. To the element $H=H(z) \in \Gamma_{\hat{\mu}}^{0}(\mathcal{S})$ associate the $C^{\infty}$ differential form

$$
\psi(z)=y^{-k} \bar{H}(z) d \bar{z} ;
$$


then for any $T \in \mathcal{G}$,

$$
\psi(T z)=\Delta_{T^{-k}}|c z+d|^{2 k} y^{-k} \overline{\boldsymbol{\rho}}(T, z)^{-1} \lambda(T) \bar{H}(z) d \bar{z}=\mu(T, z) \psi(z) .
$$

Note further that for the differential forms $\psi(z)$ and $\theta(z)=H(z) d z$ the inner product (29) has the value

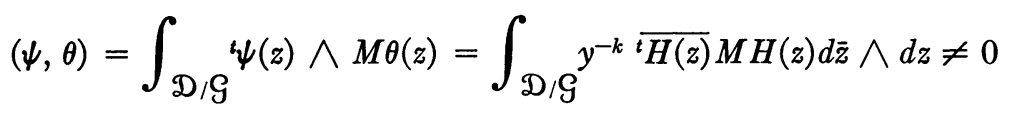

since $M$ is positive definite, the convergence of the integral following as in [11] since $H(z)$ is a cusp form. To complete the proof it clearly suffices to show that there is an element $G=G(z) \in \mathcal{G}_{n}^{0}$ such that $\bar{\partial} G=\psi$, for then $\left.G\right|_{\mu} T$ $-G=F(T) \in Z_{\mu}^{1}\left(\mathcal{G}, \mathfrak{A}_{n}^{0}\right)$ and $(F(T), H)=(\psi, \theta) \neq 0$. For this purpose introduce the iterated indefinite integrals $G_{r}(z)=(1 / r !) \int_{z_{0}}^{z}(z-t)^{r} H(t) d t$; it follows directly that $G_{r}(z) \in \mathfrak{Y}_{n}^{0}$ and that $d G_{r} / d z=G_{r-1}$ if $r>0$ and $d G_{0} / d z=H$. Consequently, letting $s=-k \geqq 0$ and $G_{-1}(z)=H(z)$,

$$
\bar{G}(z)=\sum_{r=0}^{s} \frac{s !}{(s-r) !} i^{r} y^{s-r} G_{r}(z) \in \mathfrak{S}_{n}^{0}
$$

and

$\frac{\partial \bar{G}}{\partial z}=\sum_{r=0}^{s} \frac{s !}{(s-r) !} i r\left\{y^{s-r} G_{r-1}(z)-i(s-r) y^{s-r-1} G_{r}(z)\right\}=y^{s} G_{-1}(z)=y^{-k} H(z) ;$

so that

$$
\bar{\partial} G(z)=\frac{\partial G}{\partial \bar{z}} d \bar{z}=y^{-k} \bar{H}(z) d \bar{z}=\psi(z)
$$

which concludes the proof.

It should be noted that the condition $k \leqq 0$ is really not a restriction at all, for if there exist any nontrivial functions $H(z) \in \Gamma_{\hat{\mu}}^{0}(\mathcal{G})$ then the degree of $\hat{\mu}$, which is $2-k$, must be positive, so that $k \leqq 1$; the lemma can be demonstrated to hold for the case $k=1$ as well, although the proof will not be given here $[10]$.

Lемма 7. If the kernel of $\lambda$ is of finite index in 9 and if $k \leqq 0$, where $k$ is the degree of the multiplier $\rho$, then for each nontrivial cohomology class $\{F(T)\}$ $\in H_{\mu}^{1}\left(\mathcal{G}, \mathfrak{O}_{n}^{0}\right)$ there exists a function $H \in \Gamma_{\hat{\mu}}^{0}(\mathcal{G})$ such that $(F(T), H) \neq 0$.

Proof. What is to be proved is that a one-cocycle $F(T) \in Z_{\mu}^{1}\left(\mathcal{G}, \mathfrak{A}_{n}^{0}\right)$ is cohomologous to zero if $(F(T), H)=0$ for all $H \in \Gamma_{\hat{\mu}}^{0}(\mathcal{G})$; this can be accomplished by an application of the usual method of orthogonal projection of harmonic differential forms. For this purpose let $\Lambda^{0}$ be the complex vector space of those $C^{\infty}$ vector-valued functions $F(z)$ on $D$ which vanish in an open neighborhood of each parabolic vertex of $\mathcal{G}$ and which satisfy the func- 
tional equation $F(T z)=\mu(T, z) F(z)$ for each $T \in \mathcal{G}$; and let $\Lambda^{1}$ be the complex vector space of those $C^{\infty}$ vector-valued differential forms $\phi(z)=E(z) \overline{d z}$ on $D$ of type $(0,1)$ which vanish in an open neighborhood of each parabolic vertex of $\mathcal{S}$ and which satisfy the functional equation $\phi(T z)=\mu(T, z) \phi(z)$ for each $T \in \mathcal{G}$. These are clearly pre-Hilbert spaces with the respective inner products

$$
\left(F_{1}, F_{2}\right)^{0}=\int_{D / \mathcal{G}} y^{k-2} t F_{1}(z) M \bar{F}_{2}(z) d x \wedge d y
$$

and

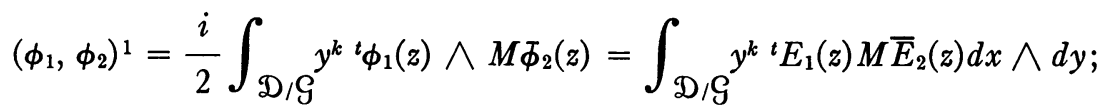

so the completions $\left[\Lambda^{0}\right],\left[\Lambda^{1}\right]$ of these spaces with respect to their inner products are then Hilbert spaces in the usual sense; these completions are the spaces of those measurable functions or differential forms respectively which are the $L_{2}$-limits of sequences of elements from $\Lambda^{0}$ or $\Lambda^{1}$ respectively. It should be noted that the subspaces of $\left[\Lambda^{0}\right],\left[\Lambda^{1}\right]$ consisting of $C^{\infty}$ elements, say $\left[\Lambda^{0}\right]^{\infty} C\left[\Lambda^{0}\right],\left[\Lambda^{1}\right]^{\infty} C\left[\Lambda^{1}\right]$, are strictly greater than the original spaces $\Lambda^{0}, \Lambda^{1}$; for an element in $\left[\Lambda^{0}\right]^{\infty},\left[\Lambda^{1}\right]^{\infty}$ need not vanish in an open neighborhood of the parabolic vertices of $\mathcal{G}$ nor even tend to zero upon approach to a parabolic vertex, though it must of course be of finite norm. The exterior differential operator of type $(0,1)$ is a linear mapping $\bar{\partial}: \Lambda^{0} \rightarrow \Lambda^{1}$, and it follows directly that the dual to $\bar{\partial}$ is the linear mapping $\bar{\partial}^{*}: \Lambda^{1} \rightarrow \Lambda^{0}$ defined by

$$
\bar{\partial}^{*} \phi(z)=\bar{\partial}^{*}(E(z) d \bar{z})=y^{2-k} \frac{\partial}{\partial z}\left(y^{k} E(z)\right) ;
$$

for if $F(z) \in \Lambda^{0}$ and

$$
\phi(z)=E(z) d \bar{z} \in \Lambda^{1}
$$

then

$$
\begin{aligned}
& (\bar{\partial} F, \phi)^{1}=\frac{i}{2} \int_{D / \mathcal{G}} y^{k} \bar{\partial}{ }^{t} F \wedge M \bar{\phi}=\frac{i}{2} \int_{D / \mathcal{G}}\left(d^{t} F\right) \wedge y^{k} M \bar{\phi} \\
& =\frac{i}{2} \int_{D / \mathcal{S}} d\left({ }^{t} F y^{k} M \bar{\phi}\right)-\frac{i}{2} \int_{D / \mathcal{G}}{ }^{t} F M d\left(y^{k} \bar{\phi}\right) \\
& =0-\frac{i}{2} \int_{D / \mathcal{G}}{ }^{t} F(z) M \frac{\partial}{\partial \bar{z}}\left(y^{k} \bar{E}(z)\right) d \bar{z} \wedge d z \\
& =\int_{D / \mathcal{G}} y^{k-2 t} F(z) M\left\{y^{2-k} \frac{\partial}{\partial \bar{z}}\left(y^{k} \bar{E}(z)\right)\right\} d x \wedge d y=\left(F, \partial^{\bar{*}_{\phi}}\right)^{0} .
\end{aligned}
$$

The composite differential operator $\Delta=\bar{\partial} \bar{\partial}^{*}$ is a linear mapping $\Delta: \Lambda^{1} \rightarrow \Lambda^{1}$, 
which is indeed a strongly elliptic differential operator at all finite points since it is of the form $\Delta(E(z) d \bar{z})=y^{2}\left(\partial^{2} E(z) / \partial \bar{z} \partial z\right)+$ (terms involving lower order derivatives). Letting $\left[\Delta \Lambda^{1}\right]$ be the closure of the linear subspace $\Delta \Lambda^{1}$ $C\left[\Lambda^{1}\right]$ and $\Psi$ be the orthogonal complement of $\left[\Delta \Lambda^{1}\right]$ in $\left[\Lambda^{1}\right]$, there results the orthogonal decomposition $\left[\Lambda^{1}\right]=\left[\Delta \Lambda^{1}\right] \oplus \Psi$. Since $\Psi$ is the set of elements $\psi \in\left[\Lambda^{1}\right]$ such that $(\Delta \phi, \psi)=0$ for all $\phi \in \Lambda^{1}$, where $\Delta$ is strongly elliptic, it follows from Weyl's lemma, in the form of Theorem $W$ of [2] for instance, that actually $\Psi$ consists of those forms $\psi \in\left[\Lambda^{1}\right]^{\infty}$ such that $\Delta \psi=0$. Moreover Lemma $M$ of [2] also holds in the present case, by using a countably infinite rather than a finite form of the diagonalization process in the proof. Therefore just as in [2] there follows an orthogonal decomposition theorem for $C^{\infty}$ forms, $\left[\Lambda^{1}\right]^{\infty}=\Delta\left[\Lambda^{1}\right]^{\infty} \oplus \Psi[10]$.

To apply this construction to the problem at hand, proving the lemma, consider firstly an element $\psi(z)=E(z) d \bar{z} \in \Psi$. Since $\bar{\partial}\left(\bar{\partial}^{*} \psi\right)=\Delta \psi=0$ it follows that $G(z)=\bar{\partial}^{*} \psi(z)$ is a holomorphic function satisfying the functional equation $G(T z)=\mu(T, z) G(z)$ for all $T \in \mathcal{G}$; but there are no nontrivial such functions when $k \leqq 0$ so that actually $\bar{\partial}^{*} \psi(z)=0$. Since then $y^{2-k} \partial / \partial z\left(y^{k} E(z)\right)$ $=\bar{\partial}^{*}(E(z) d \bar{z})=\bar{\partial}^{*} \psi(z)=0$ it follows that the function

$$
H(z)=y^{k} \bar{E}(z)
$$

is holomorphic in $D$; as in Lemma 6 this function satisfies the functional equation $H(T z)=\hat{\mu}(T, z) H(z)$ for all $T \in \mathcal{G}$, and in addition it is of finite norm in the sense of Petersson since

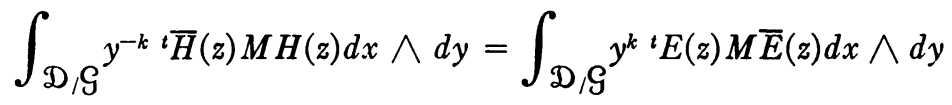

$$
\begin{aligned}
& =(\psi(z), \psi(z))^{1}<+\infty .
\end{aligned}
$$

Consequently $H(z) \in \Gamma_{\hat{\mu}}^{0}(\mathcal{S})$; so that any element $\psi \in \Psi$ must be of the form

$$
\psi(z)=y^{-k} \bar{H}(z) d \bar{z}
$$

for some $H(z) \in \Gamma_{\mu}(\mathcal{G})$. Now consider a one-cocycle $F(T) \in Z_{\mu}^{1}\left(\mathcal{G}, \mathfrak{A}_{n}^{0}\right)$ such that $(F(T), H)=0$ for all $H \in \Gamma_{\hat{\mu}}^{0}(\mathcal{G})$, and let $\phi(z) \in \Lambda^{1}$ be the differential form associated to that cocycle as in the definition of the inner product (29). Then for any differential form

$$
\psi(z)=y^{-k} \bar{H}(z) d \bar{z} \in \Psi
$$

it follows that

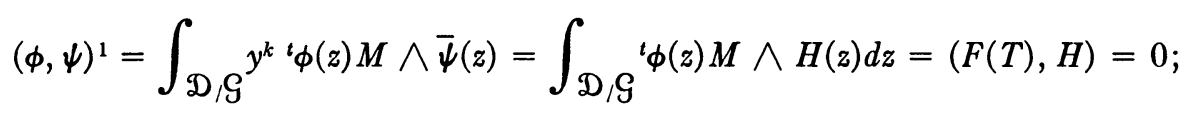

therefore by the decomposition theorem $\phi(z)=\Delta \theta(z)=\bar{\partial}\left(\bar{\partial}^{*} \theta(z)\right)$ for some 
$\theta(z) \in\left[\Lambda^{1}\right]^{\infty}$. The function $G(z)=\bar{\partial}^{*} \theta(z)$ then satisfies the functional equation $G(T z)=\mu(T, z) G(z)$ for all $T \in \mathcal{G}$, and $\phi(z)=\bar{\partial} G(z)$. Since $\phi(z)$ vanishes in an open neighborhood of each parabolic vertex of $\mathcal{G}$ the function $G(z)$ must be holomorphic in such neighborhoods; and since further $(G(z), G(z))^{0}=\left(G, \bar{\partial}^{*} \theta\right)^{0}$ $=(\bar{\partial} G, \theta)^{1}=(\phi, \theta)^{1}<\infty$, it follows indeed that $G(z) \in Z_{\mu}^{0}\left(\mathcal{G}, \tilde{\mathfrak{C}}_{n}^{0}\right)$. In view of the discussion of the inner product (29) this clearly suffices to prove that the cocycle $F(T)$ is cohomologous to zero, and thereby concludes the proof of the lemma.

It should be noted once again that the condition $k \leqq 0$ can be avoided, and is used here merely to simplify the presentation. Now the desired interpretation of the one-dimensional cohomology group follows immediately from Lemmas 6 and 7.

THEOREM 3. If the kernel of $\lambda$ is of finite index in 9 and if $k \leqq 0$, where $k$ is the degree of the multiplier $\rho$, then the dual of $H_{\mu}^{1}\left(\mathcal{G}, \mathfrak{A}_{n}^{0}\right)$ is canonically isomorphic to $\Gamma_{\hat{\mu}}^{0}(\mathcal{G})$.

5. The higher dimensional analytic cohomology groups. The cohomology groups of dimension greater than one are really the simplest of all. As a tool in the investigation of these groups consider a scalar automorphic form $f(z)$ associated to some factor of automorphy $\nu(T, z)$ for $\mathcal{G}$ on $D$; the factor of automorphy $\nu$ is of no importance here and can be quite arbitrary, but for simplicity assume that the function $f(z)$ itself has a divisor of in which each point appears with multiplicity 1 . For any $p$-cochain $F\left(T_{0}, \cdots, T_{p}\right) \in C_{\mu}^{p}\left(\mathcal{G}, \mathfrak{A}_{n}^{0}\right)$ it is clear that the product $f \cdot F\left(T_{0}, \cdots, T_{p}\right) \in C_{\mu \nu}^{p}\left(\mathcal{G}, \mathfrak{A}_{n}^{0}\right)$; hence multiplication of cochains by the element $f \in \mathfrak{A}_{1}^{0}$ defines an isomorphism $\times f: C_{\mu}^{p}\left(\mathcal{G}, \mathfrak{A}_{n}^{0}\right)$ $\rightarrow C_{\mu \nu}^{p}\left(\mathcal{G}, \mathfrak{A}_{n}^{0}\right)$, though this is of course not an isomorphism onto. Note that the point set $\delta f \subset D$ is stable under $G$, and hence associated to the factor of automorphy $\mu \nu$ is an action of the group $\mathcal{G}$ on the complex vector space $\mathfrak{\zeta}_{n}[\delta f]$ of complex-valued functions on the point set $b f$, an action defined as in (9); consequently there are cochain groups $C_{\mu \nu}^{p}\left(\mathcal{G}, \mathfrak{S}_{n}[\delta f]\right)$ and cohomology groups $H_{\mu \nu}^{p}\left(\mathcal{G}, \mathfrak{S}_{n}[\triangleright f]\right)$ defined as in $\S 2$. Furthermore the restriction of a cochain $F\left(T_{0}, \cdots, T_{p}\right) \in C_{\mu \nu}^{p}\left(\mathcal{G}, \mathfrak{A}_{n}^{0}\right)$ to the point set $\delta f \subset D$ is an element of $C_{\mu \nu}^{p}\left(\mathcal{G}, \mathfrak{S}_{n}[\delta f]\right)$; this restriction is therefore a linear mapping restr: $C_{\mu \nu}^{p}\left(\mathcal{G}, \mathfrak{A}_{n}^{0}\right)$ $\rightarrow C_{\mu \nu}^{p}\left(\mathcal{G}, \mathfrak{S}_{n}[\delta f]\right)$.

Lемма 8. The following is an exact sequence of complex vector spaces and linear maps:

$$
0 \rightarrow C_{\mu}^{p}\left(\mathcal{G}, \mathfrak{A}_{n}^{0}\right) \stackrel{\times f}{\longrightarrow} C_{\mu \nu}^{p}\left(\mathcal{G}, \mathfrak{A}_{n}^{0}\right) \stackrel{\text { restr }}{\longrightarrow} C_{\mu \nu}^{p}\left(\mathcal{G}, \mathfrak{C}_{n}[\triangleright f]\right) \rightarrow 0 ;
$$

this sequence commutes with the coboundary operators and hence gives rise to an exact cohomology sequence: 


$$
\begin{aligned}
& 0 \rightarrow H_{\mu}^{0}\left(\mathcal{G}, \mathfrak{A}_{n}^{0}\right) \rightarrow H_{\mu \nu}^{0}\left(\mathcal{G}, \mathfrak{A}_{n}^{0}\right) \rightarrow H_{\mu \nu}^{0}\left(\mathrm{G}, \mathfrak{E}_{n}[\mathrm{~b} f]\right) \rightarrow \\
& H_{\mu}^{1}\left(\mathcal{G}, \mathfrak{A}_{n}^{0}\right) \rightarrow H_{\mu \nu}^{1}\left(\mathcal{G}, \mathfrak{A}_{n}^{0}\right) \rightarrow \cdots \\
& \cdots \rightarrow H_{\mu}^{p}\left(\mathfrak{G}, \mathfrak{A}_{n}^{0}\right) \rightarrow H_{\mu \nu}^{p}\left(\mathrm{G}, \mathfrak{A}_{n}^{0}\right) \rightarrow H_{\mu \nu}^{p}\left(\mathcal{G}, \mathfrak{E}_{n}[\mathrm{~d} f]\right) \rightarrow \cdots .
\end{aligned}
$$

Proof. To show that the cochain sequence is exact only two assertions need to be verified. The first assertion is that the image of $\times f$ is the kernel of the restriction map; this is obvious, since a cochain which is in the kernel of the restriction map vanishes on of and hence is the product of $f$ and an element of $C_{\mu}^{p}\left(\mathcal{G}, \mathfrak{A}_{n}^{0}\right)$. The second assertion is that the restriction map has as image the entire space $C_{\mu \nu}^{p}\left(\mathcal{G}, \mathfrak{E}_{n}[\delta f]\right)$; this is also obvious, by considering the associated inhomogeneous cochains as in [7], and observing that any function on the point set $\delta f$ is the restriction of a function in $\mathfrak{A}_{n}^{0}$. The second half of the lemma is as obvious as its predecessor, so no further comment is needed to conclude the proof.

TheOREM 4. $H_{\mu}^{p}\left(\mathcal{G}, \mathfrak{R}_{n}^{0}\right)=0$ for all $p>1$.

Proof. First of all it is obvious that $H_{\mu \nu}^{p}\left(\mathcal{G}, \mathfrak{E}_{n}[\delta f]\right)=0$ for all $p>0$; compare [9] for instance. Therefore from the exact cohomology sequence (30) it follows that

$$
H_{\mu}^{p}\left(\mathcal{G}, \mathfrak{A}_{n}^{0}\right) \cong H_{\mu \nu}^{p}\left(\mathcal{G}, \mathfrak{A}_{n}^{0}\right)
$$

for all $p>1$, the isomorphism being that derived from the cochain homomorphism $\times f$; and this is true for any function $f$ of the type under consideration. Now to proceed by way of an argument by contradiction suppose that, contrary to what is to be proved, $H_{\mu}^{p}\left(\mathcal{G}, \mathfrak{Y}_{n}^{0}\right) \neq 0$; then let $F\left(T_{0}, \cdots, T_{p}\right) \in C_{\mu}^{p}\left(\mathcal{G}, \mathfrak{\Re}_{n}^{0}\right)$ be a nontrivial cocycle, and let $r>0$ be the dimension of the complex vector space $H_{\mu}^{p}\left(\mathcal{G}, \mathfrak{R}_{n}^{0}\right)$. For a factor of automorphy $\nu$ of suitably large degree there will be at least $r+1$ linearly independent automorphic forms $f_{i}(z)$ associated to that factor of automorphy, any linear combination of which will be a function $f(z)=\sum c_{i} f_{i}(z)$ of the type considered above; then since the map $\times f$ is an isomorphism on cohomology the cocycle $f \cdot F\left(T_{0}, \cdots, T_{p}\right)=\sum c_{i} f_{i}$ - $F\left(T_{0}, \cdots, T_{p}\right)$ must be nontrivial if not all of the constants $c_{i}$ vanish, so that the cocycles $f_{i} F\left(T_{0}, \cdots, T_{p}\right) \in C_{\mu \nu}^{p}\left(\mathcal{S}, \mathfrak{2}_{n}^{0}\right)$ must represent linearly independent cohomology classes. However since there are at least $r+1$ such, this contradicts (31), and the contradiction serves to conclude the proof.

As a final comment on this proof: by using the fact that $H_{\mu \nu}^{1}\left(\mathcal{G}, \mathfrak{C}_{n}[\delta f]\right)=0$ and interpreting the first part of the exact cohomology sequence (30) by applying the results of $\S \S 3$ and 4, there follows a Riemann-Roch theorem as in $[12 ; 6]$.

6. The polynomial cohomology groups. The final result of the present paper follows immediately upon applying the interpretations developed in 
$\S \S 3,4$, and 5 to the cohomology groups in the exact sequence of $\S 2$. As a notational convenience introduce first the factor of automorphy $\nu=\mu \cdot \delta^{1-k}$ of degree $2-k$, where $k$ is the degree of the multiplier $\rho$; note that the factor of automorphy $\hat{\mu}=\rho^{-1} \cdot \delta \cdot \bar{\lambda}$ is also of degree $2-k$, and indeed that $\hat{\mu}=\nu$ if $\lambda$ is a real representation of the group $\mathcal{G}$.

THEOREM 5. If the kernel of $\lambda$ is of finite index in $S$ and if $k<0$ then

$$
H_{\mu}^{p}\left(\mathcal{G}, \mathfrak{B}(-k)_{n}\right)=0 \quad \text { for } p>1,
$$

and there is an exact sequence of complex vector spaces and linear maps of the form

$$
0 \rightarrow \Gamma_{\nu}(\mathcal{G}) \rightarrow H_{\mu}^{1}\left(\mathcal{G}, \mathfrak{P}(-k)_{n}\right) \rightarrow \Gamma_{\hat{\mu}}^{0}(\mathcal{G}) \rightarrow 0
$$

Proof. From Theorem 2 note that $H_{\mu}^{0}\left(\mathcal{G}, \mathfrak{A}_{n}^{0}\right)=\Gamma_{\mu}(\mathcal{G})$; and since the degree of $\mu$ is $k<0$ then $\Gamma_{\mu}(\mathcal{G})=0$. From Theorem 3 note further that $H_{\mu}^{1}\left(\mathcal{G}, \mathfrak{A}_{n}^{0}\right)$ is dual to $\Gamma_{\hat{\mu}}^{0}(\mathcal{G})$ and that $H_{\mu \delta^{1-k}}^{1}\left(\mathcal{G}, \mathfrak{A}_{n}^{0}\right)$ is dual to $\Gamma_{\rho^{-1} \delta_{\lambda}}^{0}(\mathcal{G})$; again since $\rho^{-1} \delta^{k} \bar{\lambda}$ is of degree $k<0$ then $\Gamma_{\rho^{-1} \delta^{k} \lambda}^{0}(\mathcal{S})=0$ and hence $H_{\mu \delta^{1-k}}^{1}\left(\mathcal{G}, \mathfrak{A}_{n}^{0}\right)=0$. Select some isomorphism between the dual vector spaces $H_{\mu}^{1}\left(\mathcal{G}, \mathfrak{A}_{n}^{0}\right)$ and $\Gamma_{\hat{p}}^{0}(\mathcal{G})$. Equation (33) now follows by substituting these observations into the first portion of the exact cohomology sequence of Theorem 1 with $\epsilon=0$; and equation (32) follows upon combining Theorem 4 and the remainder of the exact cohomology sequence of Theorem 1 , again with $\epsilon=0$.

Note that for each subgroup $\mathcal{G}_{0} \subset \mathcal{G}$ there are the natural restriction homomorphisms $H_{\mu}^{1}\left(\mathcal{G}_{*} *\right) \rightarrow H_{\mu}^{1}\left(\mathcal{G}_{0}, *\right)$ for arbitrary coefficient groups. In particular for each parabolic transformation $T \in \mathcal{G}$ let $\{T\}$ be the cyclic subgroup of $\mathcal{G}$ generated by $T$ and consider the restriction homomorphism $H_{\mu}^{1}\left(\mathcal{S}, \mathfrak{P}(-k)_{n}\right)$ $\rightarrow H_{\mu}^{1}\left(\{T\}, \mathfrak{B}(-k)_{n}\right)$; the intersection of the kernels of these maps for all the parabolic transformations $T \in \mathcal{G}$ is a subspace $\tilde{H}_{\mu}^{1}\left(\mathcal{G}, \mathfrak{B}(-k)_{n}\right) \subset H_{\mu}^{1}\left(\mathcal{G}, \mathfrak{B}(-k)_{n}\right)$.

Corollary to Theorem 5. With the same hypotheses as in Theorem 5 there is an exact sequence of complex vector spaces and linear maps of the form

$$
0 \rightarrow \Gamma_{\nu}^{0}(\mathcal{G}) \rightarrow \tilde{H}_{\mu}^{1}\left(\mathcal{G}, \mathfrak{B}(-k)_{n}\right) \rightarrow \Gamma_{\hat{\mu}}^{0}(\mathcal{G}) \rightarrow 0 .
$$

Proof. The mapping $\alpha: \Gamma_{\nu}(\mathcal{G}) \rightarrow H_{\mu}^{1}\left(\mathcal{G}, \mathfrak{B}(-k)_{n}\right)$ in (33) is just that described in [6] associating to an automorphic form in $\Gamma_{\nu}(\mathcal{g})$ the periods of the appropriate iterated indefinite integral; and precisely as in [6] the periods associated to a cusp form represent cohomology classes in the subgroup $\tilde{H}_{\mu}^{1}\left(\mathcal{G}, \mathfrak{B}(-k)_{n}\right) \subset H_{\mu}^{1}\left(\mathcal{G}, \mathfrak{B}(-k)_{n}\right)$. The mapping $\beta$ from the kernel of $\left\{H_{\mu}^{1}\left(\mathcal{G}, \mathfrak{B}(-k)_{n}\right) \rightarrow \Gamma_{\hat{\mu}}^{0}(\mathcal{G})\right\}$ to $\Gamma_{\nu}(\mathcal{G})$ which is inverse to the mapping $\alpha$ is equally simple to describe; for if $H(T)$ is a cocycle representing a cohomology class in the kernel of $\left\{H_{\mu}^{1}\left(\mathcal{G}, \mathfrak{B}(-k)_{n}\right) \rightarrow \Gamma_{\hat{\mu}}^{0}(\mathcal{G})\right\}$ there must be an element $F \in \mathfrak{A}_{n}^{0}$ such that $H(T)=\left.F\right|_{\mu} T-F$ and then $f=\mathfrak{D}_{\rho}^{1-k} F$ is the element $\beta(H(T))$ 
$\in \Gamma_{\nu}(\mathcal{G})$. To complete the proof of the corollary it is clearly sufficient to show that $\beta$ maps a cocycle representing an element of $\tilde{H}_{\mu}^{1}\left(\mathcal{G}, \mathfrak{B}(-k)_{n}\right)$ in to the subgroup $\Gamma_{\nu}^{0}(\mathcal{G})$ of cusp forms. For this purpose consider a parabolic transformation $T$, which without loss of generality can be taken to be of the form $T: z \rightarrow z+a$ for some constant $a$. For a cocycle representing an element of $\widetilde{H}_{\mu}^{1}\left(\mathcal{G}, \mathfrak{B}(-k)_{n}\right)$ it is easy to see that $H(T)$, for $T$ as above, must indeed be a polynomial of degree at most $(-k)-1$. Then $f(z)=\beta(H(T))=d g(z) / d z$ where $g(z)=d^{(-k)} F(z) / d z^{(-k)}$ and $\left.F\right|_{\mu} T-F=H(T)$; but since $g(z) \in \mathfrak{A}_{n}^{0}$ must be such that $g(z+a)=g(z)$, it has a Fourier expansion of the form $\sum_{n=0}^{\infty} c_{n} \exp (2 \pi i n z / a)$ and consequently

$$
f(z)=\frac{d g(z)}{d z}=\sum_{n=1}^{\infty} 2 \pi i \frac{n}{a} c_{n} \exp (2 \pi i n z / a),
$$

which does show that $f(z)$ is a cusp form.

REMARKS. If $\lambda$ is a real representation of the groug $\mathcal{G}$, so that $\hat{\mu}=\nu$, it then follows from (34) that $\operatorname{dim} \tilde{H}_{\mu}^{1}\left(\mathcal{G}, \mathfrak{B}(-k)_{n}\right)=2 \cdot \operatorname{dim} \Gamma_{\mu}(\mathcal{G})$; for the trivial representation $\lambda$ this was proved in [6] simply by calculating the two dimensions. Again as in [6] the operations of the Hecke modular correspondences commute with the exact sequences (33) and (34), so that the knowledge of the eigenvalues of the Hecke operators on the polynomial cohomology groups leads to the knowledge of the Fourier coefficients of a basis for the automorphic forms associated to the group $G$.

\section{BIBLIOGRAPHY}

1. P. Appell, Sur les fonctions périodiques de deux variables, J. Math. Pures Appl. vol. 97 (1884) pp. 16-48.

2. W. L. Baily, Jr., The decomposition theorem for V-manifolds, Amer. J. Math. vol. 78 (1956) pp. 862-888.

3. G. Bol, Invarianten linearer Differentialgleichungen, Abh. Math. Sem. Univ. Hamburg vol. 16 (1949) pp. 1-28.

4. H. Cartan and S. Eilenberg, Homological algebra, Princeton, Princeton University Press, 1956.

5. M. Eichler, Modular correspondences and their representations, J. Indian Math. Soc. vol. 20 (1956) pp. 163-206. 298.

6. _- Eine Verallgemeinerung der Abelschen Integrale, Math. Z. vol. 67 (1957) pp. 267-

7. S. Eilenberg and S. MacLane, Cohomology theory in abstract groups, Ann. of Math. vol. 48 (1947) pp. 51-78.

8. L. R. Ford, Automorphic functions, New York, Chelsea, 1951.

9. R. C. Gunning, Factors of automorphy and other formal cohomology groups for Lie groups, Ann. of Math. vol. 69 (1959) pp. 314-326.

10. - A duality theorem for automorphic form, to appear.

11. H. Petersson, Über eine Metrisierung der automorphen Formen und die Theorie der Poincaréschen Reihen, Math. Ann. vol. 117 (1940) pp. 453-537.

12. J.-P. Serre, Un thérème de dualité, Comment. Math. Helv. vol. 29 (1955) pp. 9-26.

Princeton University,

Princeton, New Jersey 